This is an Accepted Manuscript of an article published by Taylor \& Francis in Journal of Further and Higher Education on 06/02/19, available online:

https://www.tandfonline.com/doi/full/10.1080/0309877X.2019.1571173 


\title{
Expanding postgraduate clinical research capacity: An exploration of key resistances
}

Simon Fletcher. 1 (corresponding author), Cheryl Whiting. 1, Annette Boaz. 1, Scott Reeves. 1

1. Faculty of Health, Social Care and Education

Kingston and St Georges University London

$6^{\text {th }}$ Floor Hunter Wing

St Georges Hospital

Cranmer Terrace

London

SW17 ORE

Simon.fletcher@sgul.kingston.ac.uk

\begin{abstract}
There have been increasing calls in healthcare for the development of a more robust evidence base. Facilitating research activity amongst clinicians is the primary means of achieving this, although engagement is often undermined by a number of barriers and resistors. This paper identifies and explores the forms of resistance which graduates from three postgraduate healthcare education programmes have encountered on their return to practice. This study employed a collective case study approach and gathered data from 29 semi structured interviews. Thematic analysis revealed a range of challenges, both anticipated and unexpected, which related to research engagement. Four forms of resistance were subsequently identified. These included: managerial, medical, organisational and interprofessional. In exploring these forms of resistance it became apparent that barriers to research engagement are not only contextually determined, but also rooted in enduring social perceptions, role insecurity and professional protectionism. The study also found that whilst research engagement was rhetorically supported, organisations offer very little tangible assistance to potential clinical researchers. A particular type of education has proved manifestly disruptive in this instance, and this disruption will need to be recognised as curricula are adjusted and developed. Further exploring the identified miscommunication between education and practice will also be of particular value to both fields.
\end{abstract}

Keywords: Postgraduate education, clinical research, resistance, professional disruption

\section{Introduction}

The last two decades has seen increasing demand in contemporary healthcare contexts for the facilitation and continuity of evidence-based practice (e.g. Aarons et al, 2009, Majid et al, 2011, Mitchell et al, 2015, Walshe and Rundall, 2001). As a result, the accredited preparation of clinical staff to undertake research has become a strategic priority in UK healthcare (AUKUH, 2014, 2016). Although the provision and quality of such courses grows year on year, there remains strong evidence to suggest that applying the research skills developed through formal education is proving to be difficult for many graduates (Logan et al, 2016, Murray et al, 2014).

Undertaking research in any context is challenging, yet attempting to engage whist practicing in clinical environments present a complex range of barriers. Harris et al (2010) offer one example of this. Exploring nursing perspectives, they found that many practitioners perceived research as 'extracurricular' or even indulgent activity, which took them away from the more important work of 
delivering patient care. Walker (1994) also found tensions between clinical practice and research engagement. Drawing attention to the continuity of established clinical roles, Walker (1994) reported that participation in research was dependent on a notional status - over-and-above the clinical role. Both these studies suggest an inherent imbalance between undertaking (higher status) clinical work and engagement in (lower status) research work.

There are other more obvious barriers to research, such as time, lack of funding and/or differing organisational policy priorities (e.g. Dilts \& Sandler 2006, Haynes \& Haines 1998) as well as more subtle, cultural constraints. In relation to the latter issue, Sitzia (2002) found that an "important factor in nursing 'culture' is that of lack of authority. Lack of authority to make change..." (p.234). As a result, the introduction of a more research intensive culture may be difficult to achieve. Similar findings have been reported in other studies (e.g. Bryar et al. 2003, Hutchinson \& Johnston 2006), which have found that cultural elements have created a number of challenges to adopting more evidence based approaches.

Thomson (2003) expressed the need to address cultural context and improve collaboration in order to foster research in nursing and other health professions (e.g. physiotherapy, occupational therapy, speech and language therapy). For Thomson (2003), "in terms of research, fostering a cultural system in which the various parts of the organization are all working to a common end can only be to its advantage" (p.144). Whilst this sets out a positive agenda of professional cohesion, as presented above, research in this area has revealed that there are numerous factors at work which not only disrupt the development of a culture of shared experience but also directly counteract it.

The challenges which face clinicians who wish to undertake research are well documented. Sung et al (2003) have identified a myriad of institutional, financial, cultural and regulatory difficulties with clinical research in American contexts. There have also been parallels reported in the United Kingdom, as Koshy and Clark (2016) explored equivalent complexities associated with (medically based) clinical trial processes. Whilst this refers to challenges in medical research, an area which has been historically prioritised over non-medical investigation, the implications for clinically driven research, largely undertaken by nurses or allied health professionals, are therefore more acutely concerning. Smith and Boyd (2012) have explored the professional transition from clinician to lecturer which a number of former nurses, midwives and allied health professionals have undergone. Whilst they were able to transition relatively easily, and were well supported in many aspects, there were also reports that mid-career changes offered particular challenges, and that it was difficult for practitioners to leave their clinical identities behind in exchange for that of an academic. This begins to offer insight into the realities for clinicians who wish to undertake research.

Martin et al (2009) have explored tensions between managers and clinicians within a general practice (GP) setting, against broader shifts in the healthcare workforces who undergo a 'modernisation' project. Describing the creation of new GP roles, there are parallels with the tensions which emerge between clinical managers and their staff members who return to practice from a time away with new qualifications and skills. Whilst their difference in level (i.e. clinician, manager) will have once been understood and accepted, broadly contributing to the continuity of the profession, modernization processes, such as increasing research engagement in clinical staff can 
disrupt these established roles. New knowledge and aspiration in clinicians who are keen to undertake research may combine with increasing managerial insecurity and this could serve to widen the divide between professionals who would have at one point been stable colleagues who were aware of their respective roles and positions.

A (2008) paper from Pickstone et al explored capacity building for research activity in allied health professionals (AHP), and in discussing the conditions needed in order to facilitate research engagement the study has served to foreground one of the most enduring restrictors for contemporary practitioners. Stating (p. 63) that:

In order to participate as equal partners, many AHPs may need research training, and this has formed a key strand of the research capacity building strategy. However, while important, training alone will not deliver sufficient gains in capacity unless it is matched with management support and funding to disseminate ideas effectively.

This succinctly identifies the way in which barriers to research go beyond the immediately measurable. (Lack of) Managerial support represents a key area of interest to this study and through exploring the professional dynamics which exist when clinicians return to practice, it will become possible to further interrogate a complex practical context.

This paper presents an analysis from a study that explored the experiences of graduates from three postgraduate courses in clinical research and improvement science, after they had returned to clinical practice. We explore the challenges, conceptualised in this paper as differing forms of 'resistance', which the graduates encountered when attempting to transfer the knowledge and skills developed during the educational experiences back to their clinical practices.

\section{Background}

Using and exploring the notion of resistance in professional contexts is likely to be associated with a conventional sociological treatment of the term, which broadly situates resistance as a reactionary measure enacted by the 'oppressed' (Duncombe, 2002). Whilst our use represents a more literal illustration of the barriers which face clinicians who attempt to engage in research, it will be useful to acknowledge the literature which critiques and conceptualises resistance in professional contexts.

Hollander and Einwohner (2004) confront the complex, and often contradictory nature of resistance. Whilst generally aligning the term with models of opposition, they state (p. 550) that, "dichotomizing resisters and dominators (in this way) ignores the fact that there are multiple systems of hierarchy, and that individuals can be simultaneously powerful and powerless within different systems". This suggests that we should not view resistance simply as a subordinate's reaction, but a more fluid and diverse characteristic of social context. Johansson and Vinthagen (2016) develop this, commenting that, "resistance has the potential to undermine power relations, per definition. However not all resistance does succeed, at least not always, or in all aspects, but might instead reproduce and strengthen relations of dominance. This is not only due to the creation of counter forces or new oppositional alliances that explicitly try to capture the state or other entrenched power institutions, but is a more fundamental paradox of inbuilt ambivalence, complexity and even 'irrationality' within resistance." (p.418) 
It is then, important that resistance is interpreted in a way which goes beyond traditional characterisations. Through exploring an 'alternative' representation of resistance, it becomes possible to approach the more fractured reality of power dynamics in professional contexts. Sundberg et al (2015) explored power and resistance in medical education, identifying a link between resistance and professional identity. These authors found that, "resistance from teachers and supervisors to educational change and development is to a large extent based on identity issues - their identity as subject experts can be threatened by change and they also often identify more with being a clinician or a researcher than being a teacher/supervisor" (p.15). As a result, it is possible to regard resistance to change as simultaneously affirming professional independence whilst acting as a barrier to pedagogical progression. As such, it is possible to interrogate the barriers to clinical research engagement from multiple perspectives. Although this study focused on interviewing clinicians from three postgraduate courses in clinical research and improvement science, who in general, had the same intentions and aspirations, our analysis did not simply position these professionals in a conventional hierarchy. Instead they were positioned as key components in a system which is subject to a complex network of competing, complementary and arbitrary behaviours. The 'resistance' that these individuals discuss below not only reflects this complexity, but also wider uncertainties in an inherently turbulent health service context.

\section{Methods}

We adopted a collective case study approach (Stake, 1994) to inform our understanding of resistances embedded within three postgraduate courses in clinical research and improvement sciences. Collective case studies allow researchers to focus on a phenomenon across cases which have been chosen to enhance understanding. Rather than focusing on each particular case, the emphasis is on the ways in which each case uniquely illuminates specific elements of the phenomenon of interest (Stake, 1994). To help us understand the nature of resistance which the graduates from three courses encountered when attempting to transfer the knowledge and skills developed during their educational experiences to their clinical practices, we selected three different participant cases (described below). By comparing our findings across three cases, we hoped to develop a multi-faceted understanding of resistance in this context.

\section{Study context}

Participants were purposively sampled from graduates who completed either a Masters by Research in Clinical Practice (MResCP) at Kingston and St George's, University London (KUSG), the Implementation and Improvement Science MSc at King's College London or attended a two day 'Implementation Science Masterclass' organised by the National Institute for Health Research (NIHR) Collaboration for Leadership in Applied Health Research and Care South London (CLAHRC).

The course at KUSG offered an opportunity for a number of health and social care professionals (e.g. nurses, midwives, pharmacists, and allied health professionals) to undertake a fully funded postgraduate course on a full time (one year) or part time (two year) basis. The MResCP adopts a collaborative approach to education and development, providing a programme of practical and academic study to enable participants to acquire the necessary research skills for future careers in clinical research. In line with a strategy for developing clinical academic researchers (Department of Health, 2012), the programme aims to build research capacity for clinical practice. 
The MSc in Improvement Science at King's College London is a one year full time or two year part time Masters programme, and aims to equip graduates with the knowledge and expertise to improve healthcare services. Heavily aligned with enhancing evidence bases, the programme enables students to 'bridge the gap' between practice and research and whilst aimed predominantly at nurses, midwives, pharmacists and other health professionals, a clinical background is not a prerequisite.

The NIHR 'Implementation Science Masterclass' was a two day event which took place at Kings College London aimed at healthcare professionals, managers, researchers, service users and patients. Designed to offer an insight into improvement science, the workshop was comprised of lectures delivered by globally diverse experts in the field of implementation science and group work which focussed on the development of the participants' own improvement projects.

\section{Data collection and analysis}

Semi-structured telephone interviews were gathered from a sample of course participants (selected to generate a range of professions) over a six month period. Each participant undertook a telephone interview of approximately 30 to 45 minutes. They explored a range of topics, including but not limited to; motivation for undertaking the course, previous research, improvement/implementation science experience, institutional support, cultural support, individual support and barriers and facilitators to engagement.

In total, 29 interviews were undertaken with the graduates from the improvement courses and comprised of the following professions: nursing $(n=10)$, physiotherapy $(n=10)$, speech and language therapy $(n=3)$, dietetics $(n=2)$, occupational therapy $(n=2)$, counselling $(n=1)$ and policy analysis $(n=1) .14$ Participants undertook the MResCP at St Georges, 7 were from the MSc in Improvement Science at Kings and the remaining 8 took part in the Improvement Science Masterclass, also at Kings. The total pool of potential participants was 78 , so the sample represented $37 \%$ of this.

The interviews were undertaken by SF who has a background in qualitative sociology and interprofessional healthcare research. The interviews built on findings which emerged from a previous pilot study (Fletcher et al, 2017). The pilot revealed a number of areas in which further investigation was warranted. These largely centred around resistance to engagement and provided the motivation to develop this research over a multiple institutional sample.

A thematic analysis of the interview transcripts was undertaken. Using an interpretation of work by Nowell et al (2017), in which thematic analysis takes on six distinct phases, we were able to safeguard the rigour and relevance of the study. Phase one saw a familiarization with the data, as the research team collectively read through the interview transcripts. Phase two saw the generation of initial codes. These early codes included Organisational Issues, in which time, resources and practical elements of context were explored. Managerial relationships, whereby we were able to initially identify the disruption which was caused by increasing qualifications and knowledge bases of clinicians. Professional hierarchies, in which medical dominance became apparent and interprofessional issues, where the professional dynamics and tensions between various disciplines was explored. This stage enabled the early identification of barriers to research engagement after 
course completion and the various associated forms of resistance. This was followed by phase three, which used the results from the coding exercise to search for more robust themes. These themes were then reviewed in phase four and named in phase five, contributing finally to the production of the report and the identification of the four key forms of resistance. By following a process such of this it becomes possible for author, reader and participant to gain insight into the systematic nature of evidence mapping, thereby ensuring a more credible study (Lincoln and Guba, 1985).

\section{Ethical considerations}

The study was approved by Faculty of Health, Social Care and Education, Kingston University and St George's University London Research Ethics Committee.

\section{Results}

Analysis of the data generated four key themes related to resistance of research knowledge and skills in clinical contexts for this sample of graduates.

\section{Managerial resistance}

The first form of resistance was linked to a tension between the course graduates and their clinical managers. Manifested in instances of 'intellectual encroachment', the new knowledge bases of returning graduates proved deeply unsettling for their clinical managers, who were forced to reassess the hierarchies which had previously sustained professional relationships. This led to the overt creation of barriers to engagement in clinical research:

From what she (manager) said, she was worried that if I became more involved in research that could perhaps lead to a promotion over her (Physio \#1)

There can be resistance definitely within certain professions if your manager isn't research active then that's very difficult. Like I've had to experience a lot of barriers like when I did my MRes, my manager did everything in her human power to stop me doing it, she didn't want me to do it at all and it was only for the fact that I went to a different MDT member who was research active was the only reason I got it (Nurse \#3)

There are considerable implications for notions of role here, as definition becomes blurred by the insecurities which 'new' qualifications evoke. There also appears to be potential for ideological divisions to open up between managers and clinicians. The apparent binary nature of being 'research active' or not creates an environment which not only stifles clinical research from the outset but also engages new forms of identity politics amongst these professionals.

In addition, informants described the combination of myriad pressures which deprioritised research engagement from a managerial perspective. Many noted that it was difficult for their managers to allocate time and resources for research against the need to demonstrate acute improvements. As outlined below:

it's becoming increasingly difficult to persuade managers to do anything that isn't ... that doesn't show, essentially, a financial benefit for the organisation, it's very difficult to get them to support (Physio \#2)

If I was to go to my boss and say effectively that I was going to spend thirty percent of my time doing research work, I think I would probably not get supported to do that (Physio \#5) 
Not only did data suggest a disparity between managers and clinicians linked to research, there was also a broader professional problem related to specialism:

There's a barrier I think especially in the NHS and in clinical practice between management and research, so there'll be clinical specialists, and then there's managers so it all depends, and that was my experience, the managers in the NHS didn't get involved in research but the clinical specialists did (SLT \#1)

As noted in the quote above, this positon presented a relatively difficult terrain for clinicians who still remained dependant on the individual inclinations of their respective managers:

Also the manager who said yes to me going for the MRes has subsequently left and been replaced by a different manager. And I can see very clearly how its gonna (sic) be much more difficult to convince this subsequent manager, so obviously that suggests that there's something about individual's attitudes (OT \#1)

In addition, there were reported failures, at various levels, to acknowledge the processes behind and value of research engagement. Physio \#2 found:

It's like you take care of patients and that's it. And the people that develop the services are usually managers, often non-clinical, who come in and make a change which is dependent on a political and social climate. Not on experience and knowledge and understanding from the clinical area

This was echoed by nurse \#3, who commented:

Because the structure and the management isn't educated enough to understand the value of research and the value of service development through research, then they'll never understand that there's a gap and that that money is an investment. I do believe that my trust is striving for better care for patients but there's a lot of short sightedness and a lack of information

The language used in the quote above provides an image of stark division between managers and clinicians who wish to undertake research. The contention that the management isn't educated enough to understand the value of research contributes to the continuity of the very attitudes and perceptions which encourage professional insecurity and the blurring of role definition on both sides. The clinicians believe what the managers fear, further widening an already significant gulf.

\section{Medical resistance}

This form of resistance was linked to the notion that medical research was regarded as having higher symbolic valuable than the research generated from other professions (e.g. nursing, occupational therapy). One informant described the way in which infrastructure is predisposed to facilitate medical dominance in clinical research:

Most of the people leading and doing clinical research in healthcare are doctors, they've got it quite well set up, it's almost part of their normal clinical training to be involved in some shape or form in clinical research and many of them take time out to do Masters or PhD as part of their clinical training, and that's supported financially and also supported to allow them to maintain their clinical contacts whilst doing their research (Nurse \#3) 
This informant went on to state that further jurisdictional tensions stemmed from non-medical involvement:

... so I suppose to them (physicians) if they see other people coming in and trying to lead research clinically they might not be so happy about it because that's their kind of realm (Nurse \#2)

Another participant developed this notion further, stating that:

I think there is this professional silo mentality to some extent now I think that if you look at our medical colleagues some of them would be reluctant to engage in research that was genuinely multidisciplinary, because they wouldn't see it as having the kind of status that straightforward medical research gets, and they'd be concerned where it might be published (Physio \#4)

These perceptions around medical colleagues are, whilst likely grounded in truth, further responsible for the forms of reactionary professional isolation which are commonplace in multidisciplinary healthcare contexts. The admission that a silo mentality exists is reflective of the simultaneous production of barriers through practical professional insularity and the subsequent perception based retreat into the comfort of the familiar and collegiate.

The data also suggested that these attitudes remained in place when research engagement was eventually obtained:

I think in non-medics there's a culture of being involved in research but being involved as a research nurse rather than a chief investigator, I think I'm always questioning why aren't you giving opportunities to someone else and it'll be like 'well the doctor's the chief investigator and I'm the research nurse' and we're like 'well why are you researching their practice and not yours' and it led me to question that a bit (Nurse \#2)

This data extract succinctly combines cultural and practical constraints, as funding has and will likely continue to be allocated in a way which is consistent with this disparity.

\section{Organisational resistance}

This form of resistance, in relation to a lack of time and resources to undertake research alongside clinical commitments also featured heavily in the graduates' dialogue, as the following quote indicated:

it's not in my job description to undertake research as it were, building a research culture yes that's part of my job, but I must say I don't have any set time to do that (OT \#3)

This quote succinctly encapsulated the frustrations which faced these graduates as new researchers, and highlighted how organisational support for them to act as researchers was lacking. Being expected to undertake research activities and develop a research culture without support was also echoed in the statement below:

if I'm meant to be doing this (research) in the same hours that I'm meant to be doing my clinical role, I have been given NO time, NO resources, NO change to my pay structure, NO change to my job description, NO change to my banding, and NO allowance of my caseload or duties in order to be able to do the research project. 
[Since returning to clinical practice] nothing has changed in my job description and although the personal annual reviews might set me goals where l'd engage in more research activity, there's nothing been changed about my job plan in order to allow that to happen, and no targets or goals have been set by anyone (Physio \#2)

Another informant describes the failure of his institution to recognise his new attributes on returning to practice:

I've just spent two years away, I've got a scholarship for this, I've brought a bit of esteem to the trust, I've developed a research project that I'm now trying to get published, I sort of thought 'how can you (the trust) hep me in my career?' But its kind of fallen on deaf ears, its kind of like its not relevant to them, despite the fact that if we look at the research that could be done it will improve services and be more cost effective (Nurse \#5)

The practical constraints which are described here compound the ideological and perception based professional tensions which have also been identified. Not only do they restrict engagement in research in isolation, but they are also complicit in the production of a culture which encourages these multiple forms of resistance to operate together. The inextricable link between service provision and what is deemed valuable in these contexts invalidates research activities.

\section{Interprofessional resistance}

The fourth form of resistance which emerged in the analysis was linked to interprofessional research (involving two or more professional groups). This was viewed by participants as a type of work that could undermine the unique value attached by members of that profession. For example, if research knowledge is developed collaboratively, how much of a role did the physiotherapist or nurse have individually? As one informant commented:

I think some of the other professions, might also have, and I have come across a little bit of this with some of the AHPs, is a sense of wanting to stick up for their own profession and can lead to a kind of wariness about something that might take away the uniqueness of their profession if you like (Physio \#5)

There were also fairly rigid perceptions around professional role, which also contributed to a sense of isolation. As one of the graduates stated:

I think its other people's perception about role, some people think you know, 'physios are better at this, OTs are better at this' so that sometimes stops some people wanting to work together (OT \#2)

Another informant discussed this notion of professional isolation:

Historically we've always had a joint national conference with the dermatologists and the nursing dermatology group and its interesting because last year was the first year that they decided that they wanted their own conference and so nurses weren't able to go, and I think potentially this is going backwards rather than working towards collaborative care (Nurse \#6)

Further perceptions which sustain professional isolationism were echoed by another informant. One participant described his experience of a 'silo mentality' in clinical practice, stating: 
Even working at the hospital, you tended to work in a silo, like you work in a silo of being in a mental health hospital and even to the point of just being your ward, you kind of work in the silo of your ward so you don't ... as a ward manager l'd spread my wings and embrace other departments and other wards and things but I think generally you kind of stuck to your own and you're in a silo so I think that was the case with mental health nursing. Obviously you'd come into contact with other disciplines throughout the course of your work like the police and the council and housing and that kind of thing, but other healthcare professionals not so much (Nurse \#5)

The admission and in a sense, resignation that professionals 'stick to their own' speaks of a base level insularity in clinical settings which will does little to engage the collaborative networks which have been proven to encourage research. Further questions around why such attitudes are in place from the outset will of course be useful, however the reflection from the participant here that this is a default for many practitioners, offers a strong indication that there are entrenched concerns regarding the movement beyond and across professional boundaries.

These instances of interprofessional tension and resistance can of course be exacerbated by a sense of competition in clinical contexts. As one participant commented:

Well no one's doing it on the other side that's the whole point. There's no integration, sometimes there's an 'us and them', there's a protectionist thing, everyone's very busy. You know at the moment it's a competition, you know 'we're doing more than you are' or 'we're more hard done by' or 'we're busier'. It's fairly dysfunctional. It's the interaction amongst different groups in the same department that's not always aligned. We're always pulling for the patient, not each other (Genetic Counsellor \#1).

There was however some encouraging evidence of reflexivity within staff, who see this tension, or professional distinction as an inevitable aspect of practice, to be negotiated as their career develops:

Moving forward as a researcher, I will be encountering these situations where I need to be able to manage people with these different professional identities, both as people who are of a higher power than I am, or at some point when I become a professional researcher myself, those who are actually under me, how to negotiate those kinds of things (Nurse \#7).

\section{Discussion}

This paper presented data which reported and explored issues of resistance to engagement in research for the graduates of three educational experiences designed to increase the research capacity of clinical staff. As presented above, these informants faced various forms of resistance on their return to clinical practice. Not only were there practical barriers to research engagement such as time/space constraints and financial limitations, their research abilities were further devalued by managerial attitudes, professional isolationism and medical dominance. Below we discuss these different forms of resistance and their implications for practice..

Managerial resistance was visible in the tension between clinical managers and returning graduates, and revealed how wider instability can engender insecurity between professionals and their managers. As indicated above, traditional hierarchies can be disrupted by the previously 'subordinate' clinicians possessing new forms of knowledge and in most cases, aspirations to work beyond their clinical roles alone. The findings suggest that it is difficult for clinical managers to 
envision the longitudinal benefit of research engagement (Hanney et al, 2013) when they are both concerned about encroachment on their professional status and increasingly expected to produce acute and immediate improvements in outcome.

The way in which resistance is evident in both the behaviour of the recent graduates, as they seek developmental opportunities beyond the traditional parameters of 'role', and the more senior professionals/managers, who resist the shift towards formal qualification, provides a striking example of the dual properties of 'resistance' in clinical research contexts. Both forms not only represent the personal and individual professional experiences of practitioners who have been exposed to divergent agendas, but also a more outward looking desire to either progress their discipline, in the case of the returning graduates, or retain some form of professional identity in the case of the managers. The range of conflicting intentions consistent with the multiple realities of resistance offers support for the contention that intervention (educational and otherwise) in clinical research engagement, should acknowledge what is a fundamental complexity.

The medical resistance theme provided an insight into the perceptions which surround medical dominance in healthcare. Freidson (1970) and Witz (1992) both suggest that medical knowledge and its assumed and acknowledged superiority places medicine in an advantageous position over the division of labour in healthcare. Medical professionals can therefore attain 'true autonomy' by evaluating the work and input of others without being subject to the same scrutiny. The idea that research, the foundation of knowledge production, is less valuable when undertaken by non-medical professionals, reveals that this is a particularly enduring perception.

Medical dominance is unlikely to diminish as it is both practically and ideologically sustained (Reeves et al., 2010). This may be subconscious or implicit, yet the influence which medical knowledge carries will often overshadow contributions from other disciplines, leading to a range of insecurities around professional identity. However, instances in which medical staff sustain this, or go further and assert their own cultural superiority over clinical colleagues, for example resisting their entry into research contexts, suggests that there is some discomfort with a decreasing knowledge gap between medical and clinical professionals. This resistance therefore, can again be interpreted in two ways. It simultaneously acts as a barrier to research engagement for clinical practitioners whilst enabling the useful exposure of problematic and constraining medical parochialism. Whilst this may not overtly counteract traditional perceptions of medical dominance, it does at least, draw attention to a narrative which has been previously obscured.

In relation to organisational resistance, there have been evident shortcomings on behalf of healthcare institutions to recognise the value of research and its potential for improving outcomes and saving money over the long term (Krzyzanowska, Kaplan and Sullivan, 2011, Tunis, Stryer and Clancy, 2003). Given that there are multiple pressures on clinical managers for immediate improvements, it is perhaps possible to attribute any reluctance to embrace research as consequence of contemporary circumstance. Whilst many administrators are likely to recognise the potential of research, it simply cannot be prioritised when all implementation is expected to contribute directly to acute care. Whereas resistance is embedded here in contextual constraints, the ideological resistance which has been evident in the dialogue represents a more problematic and perhaps more persistent barrier to clinical research engagement. The changing priorities in the NHS 
should again be referred to, as they combine to obscure the potential of research practice. These forms of resistance also cover the practical constraints which restrict access to and engagement in clinical research. Whilst time, resources and space are always likely to be limited, the way in which protected research time during clinical hours is rarely, if ever, facilitated forces research to become extra-curricular, impinging on clinical work and broadening disparities.

Interprofessional resistance reflects the tendency of professional members to attempt to protect the individuality and perceived value of their particular profession in a health service which is undergoing significant transition. This is strongly consistent with the sociological work of authors such as Larson (1977) and Abbott (1988) who have discussed the notion of a professional project whereby the autonomy of a profession depends upon the support from the state to ensure its privileged position is established, secured and sustained. Healthcare professions, particularly physiotherapy, speech and language therapy, occupational therapy and nursing, are under pressure to collaborate in their research studies. Whilst professional autonomy is unlikely to be compromised by interprofessional collaboration (Salhani \& Coulter, 2009), notional identities and contested professional jurisdictions may be disrupted by this type of work (Baker et al 2010).

It is possible to suggest that these forms of professional protectionism are products of continued turbulence for the National Health Service related to ongoing reforms in structures, service delivery and commissioning. For example, the focus on patient safety in the wake of a number of high profile institutional failings, a focus on the improvement and a wider push towards patient involvement may have contributed to this shift in protectionism. Whilst it is difficult to categorically identify the implications for clinical research, the prioritisation of improved acute outcomes and a more general climate of uncertainty, presents a distinctly unsettling professional terrain. It is perhaps unsurprising that attempts to negotiate this come in the form of a retreat to the notional sanctuary of a wellestablished and familial professional group identity. Clinical research engagement may contribute to distinct forms of fragmentation, as the disruption of familiar roles which are based largely around conventional perceptions of knowledge, causes professional relationships to become more complex. As clinicians increasingly engage, or attempt to engage, in research, the long-established structures which assigned role according to predetermined level have been challenged on both practical and ideological terms.

The way in which research is rhetorically supported yet practically undermined is particularly worthy of further investigation. The comments of one participant who described 'building a research culture' as being part of her job whilst simultaneously being allocated no time, space or resources to achieve this, suggests that the everyday pressures of clinical work will, for the foreseeable future, relegate and separate clinical research. The requirement to appear conducive to research rather than actually enable engagement creates a situation in which research and clinical practice become mutually exclusive. Research will only be facilitated under obligation, and its superficial promotion is a concerning example of this. Being research active in this context is encouraged by organisational rhetoric, yet any actual attempt to undertake or engage by a full time clinical professional can be seen as an act of resistance. It is perceived as subversive, or even negligent, to commit to research engagement above and beyond clinical priorities and this once more demonstrates how resistance is so fundamentally embedded in specific cultural systems and their associated power structures. Pledging a commitment to research, just as many NHS Trusts and other clinical institutions have 
done represents a possible contradiction. By appearing conducive to research, these organisations comply with general discourses which recognise the value of engagement in evidence-based practice, however when a clinician attempts to undertake research, this can be undermined by the everyday organisational practices, attitudes and priorities which underpin many clinical contexts.

\section{Conclusions}

Our findings suggest that the introduction of formal education in research, implementation and improvement in clinical contexts will meet difficulty due to a range of interconnected and selfsustaining forms of resistance. As we reiterate these below we also attempt to offer tentative solutions.

The tensions which emerged between managers and their staff members after these professionals returned to practice with new qualifications represented a particular area of concern. Based largely around notions of professional insecurity, the way in which research polarises managers and clinicians, and is representative of an ideological divergence serves to discourage both engagement and allocation. Although these personal insecurities are not easy to counteract, by adapting what research represents and communicating its importance and efficacy, it is possible to at least begin to respond to the diversification of the workforce in more productive ways.

Medical resistance was evident in the form of notions of medical dominance or superiority when research was discussed. Whilst this is a well-documented dilemma for clinicians and all other healthcare professionals, the narratives which we uncovered enable us to problematize ideas which surround medical precedence and challenge them accordingly. The desire to engage in research from the clinicians that we spoke to in this study took place independently of any conventional, role defined 'rules' around who should be undertaking and the subsequent quality of research, and shows an organic willingness to broaden understanding.

The organisational constraints which faced the clinicians represented a difficult challenge. There were in some instances, practical issues which rendered research above and beyond clinical practice impossible, despite promises made on behalf of managers and administrators to the contrary. Although there are objective difficulties when clinicians attempt to accommodate research activity, the reluctance to recognise its value, combined with the pressure to remain theoretically open to engagement whilst coping with the logistical realties of clinical practice, inextricably connect organisational constraints with the perceptions which surround clinical research practice. This reinforces the need to change ideas surrounding clinical research activity as we begin to see an ideological trend emerging.

The interprofessional tension which was identified in the graduates' dialogue can also be linked to ideas around professional insecurity and the need for familiarity in a turbulent contemporary healthcare context. Whilst notions of competition and role encroachment are possible consequences of a culture which devalues and blocks clinical research, the naturally collaborative characteristics of research, which encourage and in many cases necessitate cross and interprofessional interaction will provide an opportunity to learn from, with and about other professionals, rather than participating in a territorial conflict which is largely rooted in ontological fear. 
What is perhaps most challenging for those clinicians who wish to engage in research in addition to and beyond their practical commitments, is the tendency for those in positions of power to sustain a narrative in which research is deemed important whilst providing no practical or substantively ideological conditions under which it can take place. This remains one area in which it is difficult to suggest a solution as a superficial accommodation of research in clinical contexts serves to obscure the nuanced difficulties which we have described above. It is then imperative that this issue is explored in more depth.

\section{Funding}

This research was funded the National Institute for Health Research (NIHR) Collaboration for Leadership in Applied Health Research and Care South London (NIHR CLAHRC South London) at King's College Hospital NHS Foundation Trust. The views expressed in this article are those of the author(s) and not necessarily those of the NHS, the NIHR, or the Department of Health and Social Care.

\section{References}

Aarons, G, et al, (2009) The impact of evidence-based practice implementation and fidelity monitoring on staff turnover: Evidence for a protective effect. Journal of Consulting and Clinical Psychology, 77(2), 270-280

Abbott, A. (1988) The system of professions: An essay on the division of expert labor. Chicago, IL: University of Chicago Press.

AUKUH (2014) Clinical Academic Careers Pathway Capability Framework for Nurses, Midwives and Allied Health Professionals. Retrieved from: file:///C:/Users/ku56412/Downloads/AUKUH-ClinicalAcademic-Careers-Capability-Framework-May-2014.pdf

AUKUH Clinical Academic Roles Development Group. (2016). Transforming healthcare through clinical academic roles in nursing, midwifery and allied health professions: A practical resource for healthcare provider organisations. Retrieved from:

http://www.medschools.ac.uk/SiteCollectionDocuments/Transforming-Healthcare.pdf

Baker L, Egan-Lee, E, Martimianakis MA and Reeves, S. (2011). Relationships of power: implications for interprofessional education. Journal of Interprofessional Care, 25(2), 98-104.

Bryar, R, et al, (2003) The Yorkshire BARRIERS project: diagnostic analysis of barriers to research utilisation, International Journal of Nursing Studies, 40, 73-84

Department of Health (2012) Developing the Role of the Clinical Academic Researcher in the Nursing, Midwifery and Allied Health Professions, Department of Health: Leeds

Dilts, D.M. and Sandler, A.B. (2006) Invisible Barriers to Clinical Trials: The Impact of Structural, Infrastructural, and Procedural Barriers to Opening Oncology Clinical Trials, Journal of Clinical Oncology, 24(28), 4545-4552

Di Luzio, G (2008) Medical dominance and strategic action: the fields of nursing and psychotherapy in the German health care system, Sociology of Health and IIIness, 30(7), 1022-1038 
Duncombe, S (2002) Cultural Resistance Reader, Verso: London

Fletcher S, Whiting C, Boaz A, Reeves S (2017) Exploring factors related to the translation of collaborative research learning experiences into clinical practice: Opportunities and tensions, Journal of Interprofessional Care, 31(4), 543-545

Freidson, E (1970) Profession of Medicine, Harper: New York

Hanney, S, Boaz, A, Jones, T, et al (2013) Engagement in research: an innovative three-stage review of the benefits for health-care performance. Southampton: National Institute for Health Research

Harris, J, Roussel, L, Thomas, T (2010) Initiating and Sustaining the Clinical Nurse Leader Role, A Practical Guide, Bartlett: Boston

Haynes, B \& Haines, A (1998) Barriers and bridges to evidence based clinical practice, BMJ, 317(7153) 273-276

Hollander, J.A, Einwohner, R (2004) Conceptualizing Resistance, Sociological Forum, 19(4), 533-554

Hutchinson, A \& Johnston, L (2006) Beyond the BARRIERS Scale: Commonly Reported Barriers to Research Use, The Journal of Nursing Administration, 36(4), 189-199

Johansson, A, Vinthagen, S (2016) Dimensions of Everyday Resistance: An Analytical Framework, Critical Sociology, 42(3), 417-435

Koshy, A and Clark, A (2016) The barriers facing medical research in the UK, British Journal of Cardiology, 23(1)

Krzyzanowska, M.K, Kaplan, R, Sullivan, R (2011) How may clinical research improve healthcare outcomes? Annals of Oncology, 22(Suppl_7), vii10-vii15

Larson, M.S (1977) The Rise of Professionalism: A Sociological Analysis, California: University of California Press

Lincoln, Y, \& Guba, E. G. (1985) Naturalistic inquiry, Newbury Park, CA: Sage

Logan et al (2016) Transition from clinician to academic: an interview study of the experiences of UK and Australian registered nurses, Journal of Advanced Nursing, 72(3), 593-604

Majid, S, et al (2011) Adopting evidence-based practice in clinical decision making: nurses' perceptions, knowledge, and barriers, Journal of the Medical Library Association, 99(3), 229-236

Martin, G.P, Currie, G, Finn, R (2009) Reconfiguring or reproducing intra-professional boundaries? Specialist expertise, generalist knowledge and the 'modernization' of the medical workforce, Social Science and Medicine, 68(7), 1191-1198

Maybury, C, et al (2018) How does the outcome of research training fellowships funded via the NHS compare with that from competitively funded fellowships from the MRC and other charities: a crosssectional retrospective survey of trainees undertaking research training in the West Midlands, BMJ Open, 8:e019630 
Mitchell et al (2015) Increasing nurse and midwife engagement in research activity, Nursing Standard, 29(23), 37-42

Murray et al (2014) Weighing up the commitment: a grounded theory of the transition from occupational therapy clinician to academic, Australian Occupational Therapy Journal, 61(6), 437-445

Nowell et al (2017) Thematic Analysis: Striving to Meet the Trustworthiness Criteria, International Journal of Qualitative Methods, 16, 1-13

Pickstone, $C$ et al (2008) Building research capacity in the allied health professions, Evidence and Policy, 4(1), 53-68

Reeves, S, Macmillan, K, van Soeren, M (2010) Leadership of interprofessional health and social care teams: a socio-historical analysis, Journal of Nursing Management, 18(3), 258-264

Salhani, D \& Coulter, I (2009) The politics of interprofessional working and the struggle for professional autonomy in nursing, Social Science and Medicine, 68(7), 1221-1228

Sitzia, J (2002) Barriers to research utilisation: the clinical setting and nurses themselves, Intensive and Critical Care Nursing, 18, 230-243

Smith, C and Boyd, P (2012) Becoming an academic: the reconstruction of identity by recently appointed lecturers in nursing, midwifery and the allied health professions, Innovations in education and teaching international, 49(1), 63-72

Stake, R.E. (1994). Case studies. In N.K. Denzin \& Y.S. Lincoln (Eds.). Handbook of Qualitative Research (pp. 236-247). Thousand Oaks, CA: Sage Publications.

Sundberg, K, et al, (2015) Power and resistance: leading change in medical education, Studies in Higher Education, 42(3), 445-462

Sung et al (2003) Central Challenges Facing the National Clinical Research Enterprise, Journal of the American Medical Association, 289(10) 1278-1287

Thomson, D.R (2003) Fostering a Research Culture in Nursing, Nursing Enquiry, 10(3), 143-144

Tunis, S, Stryer, D, Clancy, M (2003) Increasing the Value of Clinical Research for Decision Making in Clinical and Health Policy, Journal of the American Medical Association, 290(12), 1624-1632

Walker, K (1994) Research with/in nursing: 'troubling' the field, Contemporary Nurse, 3(4) 162-168

Walshe, K. Rundall, T.G. (2001) Evidence-based Management: From Theory to Practice in Health Care, The Milbank Quarterly, 79(3), 429-457

Witz, A. (1992) Professions and patriarchy, London: Routledge.

Yielder, J. and Davis, M. (2009) 'Where radiographers fear to tread: Resistance and apathy in radiography practice', Radiography, 15 (4), pp. 345-350. 
